\title{
Propagation mechanism modelling in the near region of circular tunnels
}

\author{
K. Guan $\quad$ Z. Zhong $\quad$ B. Ai $\quad$ C. Briso-Rodríguez
}

\begin{abstract}
Along with the increase in operating frequencies in advanced radio communication systems utilised inside tunnels, the location of the break point is further and further away from the transmitter. This means that the near region lengthens considerably and even occupies the whole propagation cell or the entire length of some short tunnels. To begin with, this study analyses the propagation loss resulting from the free-space mechanism and the multi-mode waveguide mechanism in the near region of circular tunnels, respectively. Then, by conjunctive employing the propagation theory and the three-dimensional solid geometry, a general analytical model of the dividing point between two propagation mechanisms is presented for the first time. Moreover, the model is validated by a wide range of measurement campaigns in different tunnels at different frequencies. Finally, discussions on the simplified formulae of the dividing point in some application situations are made. The results in this study can be helpful to grasp the essence of the propagation mechanism inside tunnels.
\end{abstract}

\section{Introduction}

An accurate characterisation of the propagation of radio waves inside tunnels is of practical importance for the design and planning of advanced communication systems. The circular tunnel shown in Fig. 1 is a realistic subway line in Spain where we have carried out several measurements. This kind of tunnel is widely used in the modern railway and subway nowadays [1]. Hence, an accurate model revealing the propagation mechanism situation in circular tunnels is highly desirable.

A great number of propagation models presented in the last four decades indicate that there exists a 'critical distance' [1, 2], usually called the break point [1-3]. The propagation characteristics including path loss, shadow fading and small-scaled fading are different before and after the break point $[2,4,5]$. Correspondingly, the field before and after the break point are defined as the near region and the far region [6], respectively.

Normally, the distance from the transmitter to the break point is defined as [1]

$$
Z_{\mathrm{NR}}=\operatorname{Max}\left(\frac{a^{2}}{\lambda}, \frac{b^{2}}{\lambda}\right)
$$

where $a, b$ and $\lambda$ denote the width and the height of the equivalent rectangular tunnel, as well as the signal wavelength in metres. In the case of the circular tunnel, the location of the break point can be given by a special type of (1)

$$
Z_{\mathrm{NR}}=\frac{(2 R)^{2}}{\lambda}
$$

where $R$ is the radius of the cross-sectional circle. Please note, $Z_{\mathrm{NR}}$ is inversely proportional to the wavelength.

By making a brief review on the advancement of radio communication systems, we can find that the near region inside tunnels lengthens considerably owing to the increase in operating frequencies. In the public communication area, the typical systems can be roughly listed as global system for mobile communications, third generation, wireless fidelity and world interoperability for microwave access. Their frequencies are $900 / 1800 \mathrm{MHz}, 2 \mathrm{GHz}, 2.4 / 5 \mathrm{GHz}$ and $3.8 \mathrm{GHz}$, respectively. Assuming that the maximum of the radius of the circular tunnel is $5 \mathrm{~m}$, which is very common for the double track subway tunnel or railway tunnel all over the world, (2) deduces the length of the near region in each system as $300 / 600,666.7,800 / 1667$ and $1267 \mathrm{~m}$, respectively. Similarly, in the field of the dedicated communication, the list can be: terrestrial trunked radio [7], global system for mobile communications for railway [8], communication-based train control system (CBTC) [9] and dedicated short-range communications (DSRC) [10]. Their operating frequencies are $400 \mathrm{MHz}, 900 \mathrm{MHz}, 2.4 \mathrm{GHz}$ and $5.8 / 5.9 \mathrm{GHz}$, respectively. The corresponding near regions can be calculated as $133.3,300,800$ and $1933 / 1967 \mathrm{~m}$, respectively. Apparently, this great change reveals the fact 


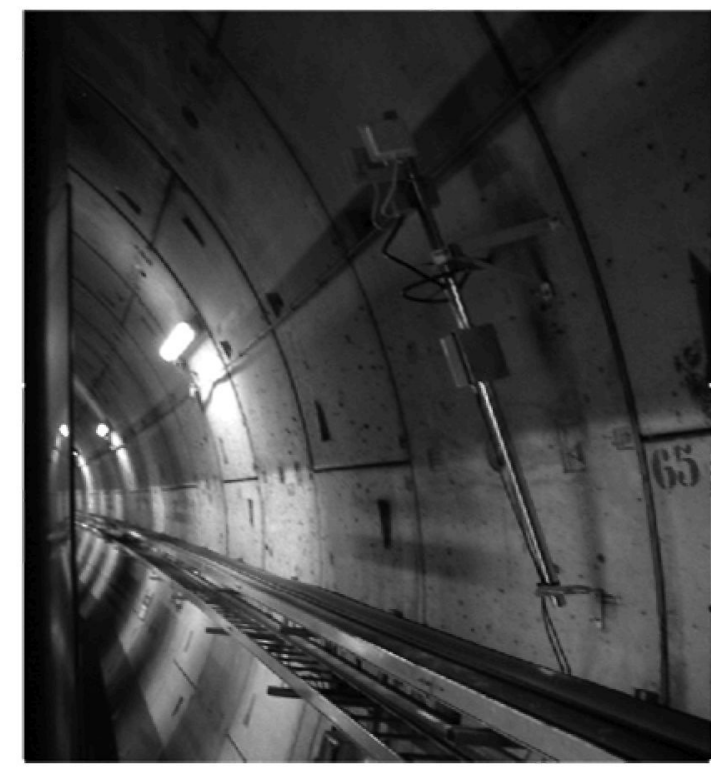

Fig. 1 Photo of a realistic circular tunnel in Madrid, Spain

that the near region may occupy most of the propagation cell. Particularly, in some short tunnels or high reliable systems that require a moderate overlap of the transmitters, the entire propagation cell could even only be in the near region. This requires that the designers of advanced radio communication systems in tunnel environments have to grasp the propagation mechanism situation in the near region.

The propagation characteristics in the far region are clear, which follow the fundamental-mode waveguide mechanism $[1,2,6,11]$. However, there is no unanimous consensus yet on the propagation in the near region. Some dissertations deem that the propagation before the break point should be interpreted by the single ray (free-space) theory $[6,11,12]$, others are inclined to advocate that it should be described by the multi-mode waveguide model $[1,2,13]$. In fact, a great deal of evidence proves that both propagation mechanisms exist simultaneously. Hence, the traditional way of interpreting the propagation in the near region by a single mechanism limits the prediction accuracy. Take the simulcast radio communication system for instance, only using the free-space propagation model can overestimate the path loss in the near region, and therefore results in an excessive overlap between neighbour transmitters. This brings large interference and reduces the efficiency of the whole system. If we predict the propagation only with the multi-mode waveguide model, the transmitter can be overmuch far away from each other, and fainting can happen between the transmitters. Thus, in order to handle this problem and reveal the propagation mechanism situation in the near region it is essential to model the accurate location of the dividing point between these two mechanisms.

\section{Analytical modelling of the propagation mechanisms and their dividing point}

\subsection{Geometrical and electrical modelling for the tunnel}

As shown in Fig. 2, from a theoretical point of view, a circular tunnel can be regarded as an oversized imperfect hollow

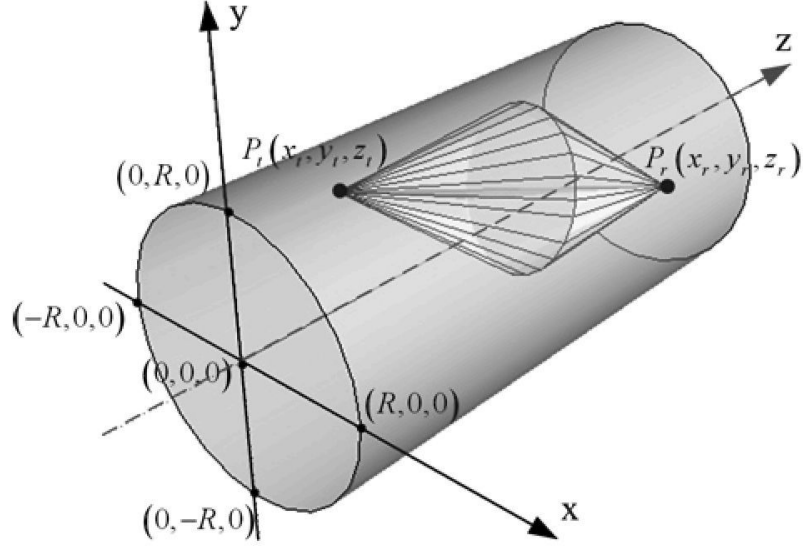

Fig. 2 Schematic diagram of the propagation inside circular tunnels with the first Fresnel zone clearance

circular waveguide. In order to use the geometrical and modal analysis, the following parameters are required:

- A coordinate system: a three-dimensional (3D) Cartesian coordinate system, with its origin located at the centre of the cross-sectional circle at the starting point of the relevant tunnel segment.

- Geometric dimensions: the radius of the cross-sectional circle is $R$. The coordinates of the transmitter, the receiver and the middle point on the line of sight between transmitter and receiver are $P_{\mathrm{t}}\left(x_{\mathrm{t}}, y_{\mathrm{t}}, z_{\mathrm{t}}\right), P_{\mathrm{r}}\left(x_{\mathrm{r}}, y_{\mathrm{r}}, z_{\mathrm{r}}\right)$ and $P_{0}\left(x_{0}, y_{0}, z_{0}\right)$. According to the $3 \mathrm{D}$ geometry theory, the relationships among them are expressed by

$$
x_{0}=\frac{x_{\mathrm{r}}+x_{\mathrm{t}}}{2}, \quad y_{0}=\frac{y_{\mathrm{r}}+y_{\mathrm{t}}}{2}, \quad z_{0}=\frac{z_{\mathrm{r}}+z_{\mathrm{t}}}{2}
$$

- Electrical parameters: relative permittivity for the wall of circular tunnel: $\varepsilon_{\mathrm{r}}$, with the typical values for concrete: $\varepsilon_{\mathrm{r}}=5[1]$.

\subsection{Propagation loss owing to different propagation mechanisms}

- Propagation loss in the free-space propagation segment. In the adjacent region of the transmitter antenna, the angles of incidence from the ray to the wall are high, resulting in high attenuation of reflected rays, whereas the path difference between direct and reflected rays may also cause additional attenuation. Therefore only the direct ray significantly contributes to the strength of the received signal. The channel loss in this segment follows the freespace loss attenuation [14]

$$
\operatorname{PL}(\mathrm{dB})=-10 \log _{10}\left[\frac{\lambda^{2}}{(4 \pi)^{2}\left|z_{\mathrm{r}}-z_{\mathrm{t}}\right|^{2}}\right]
$$

where $\left|z_{\mathrm{r}}-z_{\mathrm{t}}\right|$ is the distance between the transmitter and the receiver in metres.

- Propagation loss in the multi-mode waveguide segment. Since the Ultra-High Frequency (UHF) is considerably higher than the cutoff frequency of the fundamental modes, which is very low (i.e. some megahertz), a wide range of $E_{\mathrm{mn}}$ multiple modes propagate inside circular tunnels when 
the free-space segment ends [11]. Using the modal theory, the general expression of the attenuation constant with horizontally and vertically polarised $E_{\mathrm{mn}}$ modes inside various tunnels [15], such as circular tunnel, rectangle tunnel, arch tunnel, oval tunnel and so on, can be written as

$$
\begin{aligned}
& \alpha(m, n)^{h}=\rho \lambda^{2}\left(\frac{m^{2} \varepsilon_{\mathrm{r}}}{w^{3} \sqrt{\varepsilon_{\mathrm{r}}-1}}+\frac{n^{2}}{h^{3} \sqrt{\varepsilon_{\mathrm{r}}-1}}\right) \mathrm{dB} / m \\
& \alpha(m, n)^{v}=\rho \lambda^{2}\left(\frac{m^{2}}{w^{3} \sqrt{\varepsilon_{\mathrm{r}}-1}}+\frac{n^{2} \varepsilon_{\mathrm{r}}}{h^{3} \sqrt{\varepsilon_{\mathrm{r}}-1}}\right) \mathrm{dB} / m
\end{aligned}
$$

where $w$ and $h$ denote the max of the width and the height, respectively; $\rho$ is a coefficient, its value varies by the different shape of the tunnel. To the circular tunnel, the parameters offered above are $w=h=2 R, \rho=5.09$. Hence, the losses of horizontally and vertically polarised $E_{\mathrm{mn}}$ modes inside circular tunnels can be given by

$$
\alpha(m, n)^{h}=5.09 \lambda^{2}\left(\frac{m^{2} \varepsilon_{\mathrm{r}}}{(2 R)^{3} \sqrt{\varepsilon_{\mathrm{r}}-1}}+\frac{n^{2}}{(2 R)^{3} \sqrt{\varepsilon_{\mathrm{r}}-1}}\right) \mathrm{dB} / m
$$

$$
\alpha(m, n)^{v}=5.09 \lambda^{2}\left(\frac{m^{2}}{(2 R)^{3} \sqrt{\varepsilon_{\mathrm{r}}-1}}+\frac{n^{2} \varepsilon_{\mathrm{r}}}{(2 R)^{3} \sqrt{\varepsilon_{\mathrm{r}}-1}}\right) \mathrm{dB} / m
$$

With these propagation constants, the propagation loss in the multi-mode waveguide segment can be calculated considering losses of modes for both polarisations as

$$
L_{\mathrm{mn}}^{v / h}(\mathrm{~dB})=10 \log \left[\sum_{i=1}^{m} \sum_{j=1}^{n} \times \sqrt{10^{2 \alpha(i, j)^{h}\left|z_{\mathrm{r}}-z_{\mathrm{t}}\right|}+10^{2 \alpha(i, j)^{v}\left|z_{\mathrm{r}}-z_{\mathrm{t}}\right|}}\right]
$$

\subsection{Dividing point between different propagation mechanisms}

In order to grasp the accurate propagation mechanism situation, it is essential to model the location of the dividing point between the free-space propagation segment and the multi-mode waveguide segment. Fig. 3 depicts the 3D geometry schematic diagram of the circular tunnel, transmitter, receiver, line of sight and the maximum first Fresnel zone. According to the 3D solid geometry, the surface of the circular tunnel is the set of points whose coordinates $(x, y)$ satisfy the following equation

$$
x^{2}+y^{2}=R^{2}
$$

with $z$-coordinates completely arbitrary. The maximum Fresnel zone plane can be expressed by a plane in a general type

$$
\begin{aligned}
& \left(x_{\mathrm{r}}-x_{\mathrm{t}}\right)\left(x-\frac{x_{\mathrm{r}}+x_{\mathrm{t}}}{2}\right)+\left(y_{\mathrm{r}}-y_{\mathrm{t}}\right)\left(y-\frac{y_{\mathrm{r}}+y_{\mathrm{t}}}{2}\right) \\
& +\left(z_{\mathrm{r}}-z_{\mathrm{t}}\right)\left(z-\frac{z_{\mathrm{r}}+z_{\mathrm{t}}}{2}\right)=0
\end{aligned}
$$

The intersection between the maximum Fresnel zone plane and

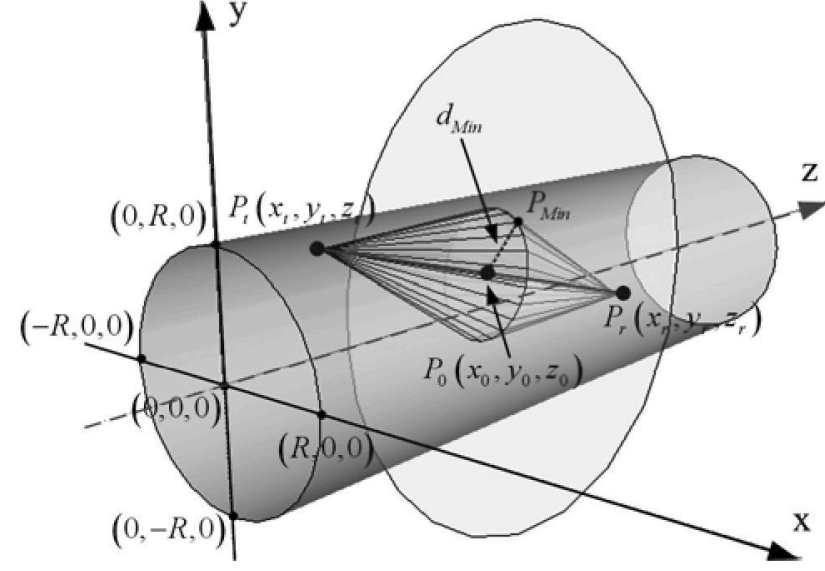

Fig. 3 Detailed schematic diagram of the propagation inside circular tunnels with the first Fresnel zone clearance

the surface of circular tunnel is a curve, which can be written as

$$
\left\{\begin{array}{l}
\left(x_{\mathrm{r}}-x_{\mathrm{t}}\right)\left(x-\frac{x_{\mathrm{r}}+x_{\mathrm{t}}}{2}\right)+\left(y_{\mathrm{r}}-y_{\mathrm{t}}\right)\left(y-\frac{y_{\mathrm{r}}+y_{\mathrm{t}}}{2}\right) \\
\quad+\left(z_{\mathrm{r}}-z_{\mathrm{t}}\right)\left(z-\frac{z_{\mathrm{r}}+z_{\mathrm{t}}}{2}\right)=0 \\
x^{2}+y^{2}=R^{2}
\end{array}\right.
$$

Define the first equation as a function $f(x, y, z)$ by

$$
\begin{aligned}
f(x, y, z)= & \left(x_{\mathrm{r}}-x_{\mathrm{t}}\right)\left(x-\frac{x_{\mathrm{r}}+x_{\mathrm{t}}}{2}\right)+\left(y_{\mathrm{r}}-y_{\mathrm{t}}\right) \\
& \times\left(y-\frac{y_{\mathrm{r}}+y_{\mathrm{t}}}{2}\right)+\left(z_{\mathrm{r}}-z_{\mathrm{t}}\right)\left(z-\frac{z_{\mathrm{r}}+z_{\mathrm{t}}}{2}\right)
\end{aligned}
$$

Define the second equation as a function $g(x, y, z)$ by

$$
g(x, y, z)=x^{2}+y^{2}-R^{2}
$$

To find the minimal distance between the intersection (curve) and the middle point $P_{0}\left(x_{0}, y_{0}, z_{0}\right)$ on the line of sight, the Lagrange multiplier method seeking extremum is employed. Let us construct a function as follows

$$
\begin{aligned}
F(x, y, z, \xi, \mu)= & \left(x-\frac{x_{\mathrm{r}}+x_{\mathrm{t}}}{2}\right)^{2}+\left(y-\frac{y_{\mathrm{r}}+y_{\mathrm{t}}}{2}\right)^{2} \\
& +\left(z-\frac{z_{\mathrm{r}}+z_{\mathrm{t}}}{2}\right)^{2}+\xi f(x, y, z)+\mu g(x, y, z)
\end{aligned}
$$

where $\xi$ and $\mu$ are the Lagrange multipliers. By seeking a partial derivative of $x, y$ and $z$, (15) can be transformed to

$$
\begin{aligned}
& \frac{\partial F}{\partial x}=2\left(x-\frac{x_{\mathrm{r}}+x_{\mathrm{t}}}{2}\right)+\xi\left(x_{\mathrm{r}}-x_{\mathrm{t}}\right)+\mu 2 x=0 \\
& \frac{\partial F}{\partial y}=2\left(y-\frac{y_{\mathrm{r}}+y_{\mathrm{t}}}{2}\right)+\xi\left(y_{\mathrm{r}}-y_{\mathrm{t}}\right)+\mu 2 y=0
\end{aligned}
$$




$$
\begin{gathered}
\frac{\partial F}{\partial z}=2\left(z-\frac{z_{\mathrm{r}}+z_{\mathrm{t}}}{2}\right)+\xi\left(z_{\mathrm{r}}-z_{\mathrm{t}}\right)=0 \\
\frac{\partial F}{\partial \xi}=\left(x_{\mathrm{r}}-x_{\mathrm{t}}\right)\left(x-\frac{x_{\mathrm{r}}+x_{\mathrm{t}}}{2}\right)+\left(y_{\mathrm{r}}-y_{\mathrm{t}}\right) \\
\left(y-\frac{y_{\mathrm{r}}+y_{\mathrm{t}}}{2}\right)+\left(z_{\mathrm{r}}-z_{\mathrm{t}}\right)\left(z-\frac{z_{\mathrm{r}}+z_{\mathrm{t}}}{2}\right)=0 \\
\frac{\partial F}{\partial \mu}=x^{2}+y^{2}-R^{2}=0
\end{gathered}
$$

By seeking the simultaneous solution of (16)-(20), the coordinate of intersection point with the minimal distance to $P_{0}\left(x_{0}, y_{0}, z_{0}\right)$ can be obtained: $p_{\text {Min }}\left(x\left(z_{\mathrm{r}}\right), y\left(z_{\mathrm{r}}\right), z\left(z_{\mathrm{r}}\right)\right.$. Therefore the minimal distance between $P_{0}$ and the intersection (curve) between the maximum Fresnel zone plane and the wall of circular tunnel can be expressed as

$$
\begin{aligned}
d_{\mathrm{Min}}\left(z_{\mathrm{r}}\right)= & {\left[\left(x\left(z_{\mathrm{r}}\right)-\frac{x_{\mathrm{r}}+x_{\mathrm{t}}}{2}\right)^{2}+\left(y\left(z_{\mathrm{r}}\right)-\frac{y_{\mathrm{r}}+y_{\mathrm{t}}}{2}\right)^{2}\right.} \\
& \left.+\left(z\left(z_{\mathrm{r}}\right)-\frac{z_{\mathrm{r}}+z_{\mathrm{t}}}{2}\right)^{2}\right]^{(1 / 2)}
\end{aligned}
$$

Based on the propagation theory, the radius of the first Fresnel zone is determined by

$$
r_{1}=\sqrt{\frac{\lambda d_{1} d_{2}}{d_{1}+d_{2}}}
$$

where $d_{1}$ denotes the distance between the transmitter and the interaction between the line of sight and the first Fresnel zone, $d_{2}$ denotes the distance between the receiver and the interaction. When the interaction is the middle point $P_{0}$, then $d_{1}=d_{P_{\mathrm{t}} P_{0}}=d_{2}=d_{P_{0} P_{\mathrm{r}}}=(1 / 2) d_{P_{\mathrm{t}} P_{\mathrm{r}}}$. At this point, the radius attains the maximum value of the first Fresnel zone plate

$$
r_{1 \mathrm{Max}}\left(z_{\mathrm{r}}\right)=\frac{1}{2} \sqrt{\lambda d_{P_{\mathrm{t}} P_{\mathrm{r}}}}
$$

The propagation theory indicates that the free-space loss channel model can be applied if the first Fresnel zone is free of any obstacles. Thus, the dividing point between two propagation mechanisms locates at $z_{\mathrm{r}}$, which is the minimal positive real root of the following equation

$$
r_{1 \mathrm{Max}}\left(z_{\mathrm{r}}\right)=d_{\mathrm{Min}}\left(z_{\mathrm{r}}\right)
$$

This means that the maximum first Fresnel zone first touches the wall of circular tunnels.

\section{Dividing point model validation}

A wide range of measurement campaigns published before have been used for validating the model of the dividing point presented in the previous section. The chosen tunnels possess great diversity: the tunnel type includes road tunnel, pedestrian tunnel and railway tunnel; the operating frequency involves 400,450 and $900 \mathrm{MHz}$. Relevant parameters in these measurements are cited as follows:

- Measurements in the railway tunnel [1]: $\lambda=0.33$, $R=7.6, x_{\mathrm{t}}=4.8, y_{\mathrm{t}}=1.5, z_{\mathrm{t}}=0, x_{\mathrm{r}}=2, y_{\mathrm{r}}=0$.

- Measurements in the pedestrian tunnel [11]: $\lambda=0.75$, $R=5, x_{\mathrm{t}}=0, y_{\mathrm{t}}=0, z_{\mathrm{t}}=0, x_{\mathrm{r}}=0, y_{\mathrm{r}}=0.2$.
- Measurements in the road tunnel [11]: $\lambda=0.75, R=5.28$, $x_{\mathrm{t}}=1.5, y_{\mathrm{t}}=-1, z_{\mathrm{t}}=0, x_{\mathrm{r}}=1.5, y_{\mathrm{r}}=-1$.

- First group of measurements in the vehicle tunnel [16]: $\lambda=0.66, \quad R=4.3, \quad x_{\mathrm{t}}=1.8, \quad y_{\mathrm{t}}=0, \quad z_{\mathrm{t}}=0, \quad x_{\mathrm{r}}=1.8$, $y_{\mathrm{r}}=0$.

- Second group of measurements in the vehicle tunnel [16]: $\lambda=0.33, R=4.3, x_{\mathrm{t}}=1.8, y_{\mathrm{t}}=0, z_{\mathrm{t}}=0, x_{\mathrm{r}}=1.8, y_{\mathrm{r}}=0$.

Table 1 shows the global comparisons of the dividing point inside tunnels between the results of model and the measurements. The results indicate that the model for the dividing point has a good performance in different type tunnels at various frequencies.

To depict the effect of the entire propagation model in the near region of circular tunnels, the measurement carried out in a railway tunnel in Spain at $900 \mathrm{MHz}$ [1] has been employed. As shown in Fig. 4, the dividing point clearly separates two propagation segments. The propagation in the segment before the dividing point follows the free-space mechanism, and the free-space loss model has a good agreement with the measured data. The segment after the dividing point is dominated by the multi-mode waveguide mechanism, and the multi-mode waveguide loss model shows a reasonable performance as well.

All the validation results and comparisons offered above indicate that the model of the propagation mechanisms and

Table 1 Comparisons of the dividing point inside tunnels between the model and the measurements

\begin{tabular}{lccc}
\hline Tunnel & $\begin{array}{c}\text { Frequency, } \\
\mathrm{GHz}\end{array}$ & $\begin{array}{c}\text { Measured } \\
\text { result, } \mathrm{m}\end{array}$ & $\begin{array}{c}\text { Modelled } \\
\text { result, } \mathrm{m}\end{array}$ \\
\hline railway tunnel & 0.9 & 30 & 29.07 \\
Spain [1] & - & - & - \\
pedestrian tunnel & 0.4 & 15 & 14.93 \\
Europe [11] & - & - & - \\
road tunnel & 0.4 & 15 & 14.42 \\
Austria-Slovenia [11] & - & - & - \\
vehicle tunnel & 0.45 & $10-13$ & 10.71 \\
France [16] & - & - & - \\
vehicle tunnel & 0.9 & $12-15$ & 13.77 \\
France [16] & - & - & - \\
\hline
\end{tabular}

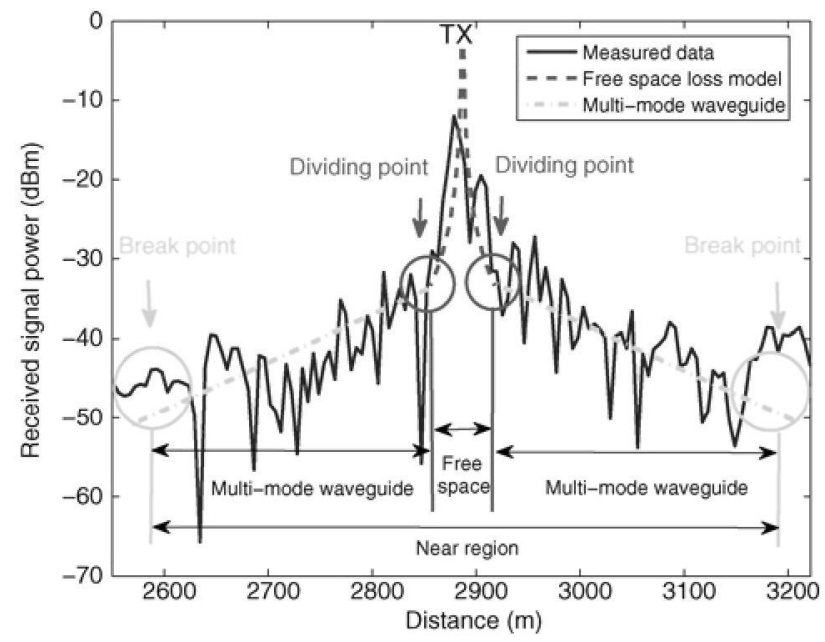

Fig. 4 Comparisons of the propagation mechanisms and their dividing points in the near region inside tunnels between measurement and theory 
their dividing point in the near region is valid and easy to use. With this model, the designer of the simulcast radio communication system can determine the proper overlap between transmitters, maximise the coverage distance, and minimise the radiated power and the interference.

\section{Dividing point model simplification in certain application situations}

In some application situations, the locations of transmitting antennas and receiving antennas, as well as the motion trajectories of mobile stations follow certain rules. In this section, the simplified formulae of the dividing point model presented in the previous section are deduced corresponding to each application situation.

\subsection{Special situation one}

In some cases, such as CBTC [9], particularly in wide tunnels, the transmitter installed on-wall and the receiver installed on-train have similar deviation on the $x$-axis, that is, $x_{\mathrm{t}}=x_{\mathrm{r}}=X, y_{\mathrm{t}}=y_{\mathrm{r}}=0$, as shown in Fig. 5 .

In this situation, the location of the dividing point is the minimal positive real root of (24)

$$
z_{\mathrm{r}}=\frac{4(R-X)^{2}}{\lambda}
$$

\subsection{Special situation two}

In some systems, such as DSRC, the communication is among different vehicles (car or carriage). In this case, the transmitter and the receiver always have similar heights: $y_{\mathrm{t}}=y_{\mathrm{r}}=Y, x_{\mathrm{t}}=x_{\mathrm{r}}=0$, as shown in Fig. 6.

By substituting the above condition into (24), the analytical formula of the dividing point can be obtained as

$$
z_{\mathrm{r}}=\frac{4(R-Y)^{2}}{\lambda}
$$

\subsection{Special situation three}

In some long tunnels, especially when the operating frequency is at several giga Hertz, the near region is very long. In this case, by using the modal theory, the transmitter and the receiver can be approximated to locate at the centre of the tunnel's cross-section: $x_{\mathrm{t}}=x_{\mathrm{r}}=X$, $y_{\mathrm{t}}=y_{\mathrm{r}}=0$, as shown in Fig. 7. By substituting the above condition into (24), the analytical formula of the distance

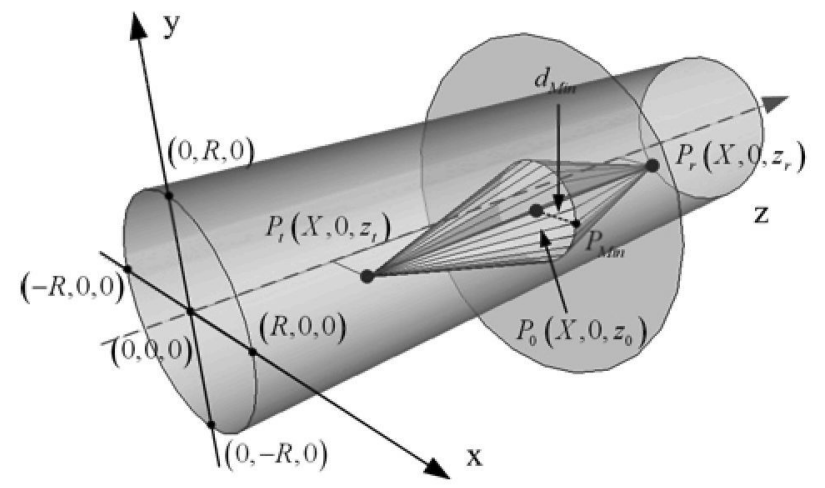

Fig. 5 Special situation one: $x_{t}=x_{r}=X, y_{t}=y_{r}=0$

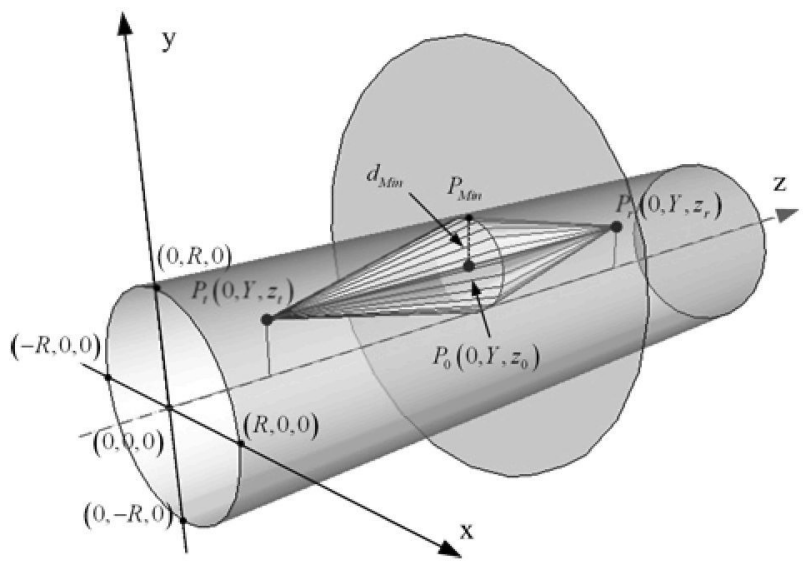

Fig. 6 Special situation two: $y_{t}=y_{r}=Y, x_{t}=x_{r}=0$

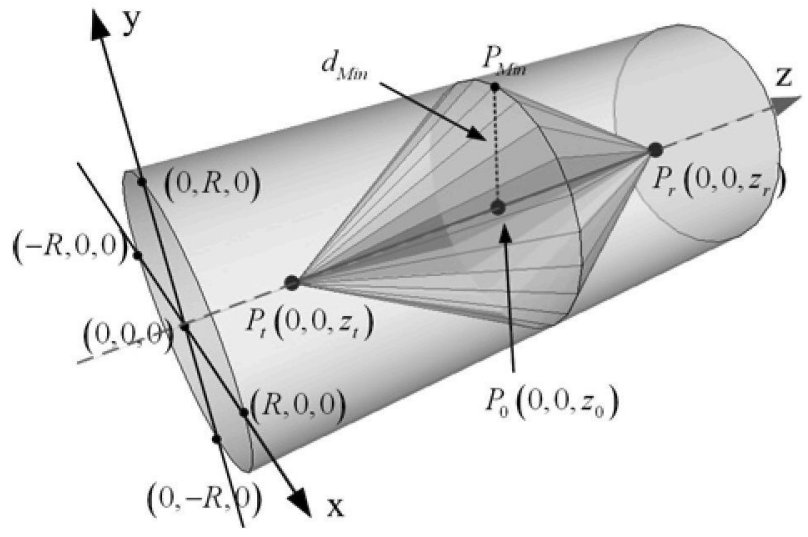

Fig. 7 Special situation three: $x_{i}=x_{r}=0, y_{t}=y_{r}=0$

where the free-space propagation mechanism ends can be obtained as

$$
z_{\mathrm{I}}=\frac{4 R^{2}}{\lambda}
$$

One interesting result is that in this case (27) is the same as (2) that is the break point separating the near region and the far region. This means that in the whole near region the propagation follows the free-space mechanism, and therefore the free-space model can be employed to predict the propagation loss in the entire near region. It is noteworthy that this conclusion is justified only in this case but not in general.

\section{Conclusions}

This paper couples previous seemingly conflicting viewpoints on the propagation mechanism in the near region of circular tunnels. A general analytical model of the propagation loss resulting from the free-space propagation mechanism and the multi-mode waveguide propagation mechanism, as well as the accurate location of the dividing point between different propagation mechanisms is presented for the first time.

This model is validated by a wide range of measurements in different type tunnels at different operating frequencies. The simplified formulae of the dividing point in some application situations render the model easy to use in 
certain realistic scenarios. The analysis, the approach and the model in this paper give heuristic explanations of the propagation mechanisms in the near region of tunnels, and therefore can help to gain deeper insight of corresponding scientific field, as well as the engineering implementations.

\section{Acknowledgments}

This work was supported by the NNSF of China under Grant no. 60830001, Program for New Century Excellent Talents in University under Grant no. NCET-09-0206, Beijing NSF 4112048 , the Key Project of State Key Laboratory of Rail Traffic Control and Safety under Grant no. RCS2008ZZ006, the Fundamental Research Funds for the Central Universities under Grant no. 2010JBZ008 and the State Key Laboratory of Rail Traffic Control and Safety (Contract numbers RCS2010ZT012 and RCS2010K008), Beijing Jiaotong University.

\section{References}

1 Briso-Rodriguez, C., Cruz, J.M., Alonso, J.I.: 'Measurements and modeling of distributed antenna systems in railway tunnels', IEEE Trans. Veh. Technol., 2007, 56, (5), pp. 2870-2879

2 Zhang, Y.P., Hwang, Y.: 'Enhancement of rectangular tunnel waveguide model'. Proc. Microwave Conf. APMC, December 1997, pp. $197-200$

3 Zhang, Y.P., Hwang, Y.: 'Characterization of uhf radio propagation channels in tunnel environments for microcellular and personal communications', IEEE Trans. Veh. Technol., 1998, 47, (1), pp. $283-296$
4 Alonso, J., Izquierdo, B., Romeu, J.: 'Break point analysis and modelling in subway tumels'. Proc. Third European Conf. on Antennas and Propagation, June 2009, pp. 3254-3258

5 Guan, K., Zhong, Zh.D., Ai, B., Briso-Rodriguez, C.: 'Research of propagation characteristics of break point, near zone and far zone under operational subway condition'. Proc. Int. Conf. on Communication and Symp. on Mobile Computing, Communication and Information Theory, Caen, France, June 2010, pp. 114-118

6 Zhang, Y.P.: 'Novel model for propagation loss prediction in tunnels', IEEE Trans. Veh. Technol, 2003, 52, (5), pp. 1308-1314

7 ETSI ETR 300-3 ed.1 (2000-02): 'Terrestrial trunked radio (TETRA); voice plus data $(\mathrm{V}+\mathrm{D})$; designers' guide; Part 3: direct mode operation (DMO)', 2000

8 http://www.uic.asso.fr, accessed August 2011

9 Rail Transit Interface Standards Committee of the IEEE Vehicular Technology Society: 'IEEE standard for communications-based train control (CBTC) performance and functional requirements', 1999

10 'Notice of proposed rulemaking and order FCC 03-324', Federal Communications Commission, 2003

11 Hrovat, A., Kandus, G., Javornik, T.: 'Four-slope channel model for path loss prediction in tunnels at $400 \mathrm{MHz}$, IET Microw. Antennas Propag., 2010, 4, (5), pp. 571-582

12 Zhang, Y.P., Zheng, G.X., Sheng, J.H.: 'Radio propagation at $900 \mathrm{MHz}$ in underground coal mines', IEEE Trans. Antennas Propag., 2001, 49, (5), pp. 757-762

13 Klemenschits, T., Bonek, E.: 'Radio coverage of road tunnels at 900 and $1800 \mathrm{MHz}$ by discrete antennas'. Proc. Fifth IEEE Int. Symp. on PIMRC, Hague, Netherlands, September 1994, pp. 411-415

14 Saunders, S.: 'Antennas and propagation for wireless communication systems' (John Wiley and Sons Ltd, Chichester, England, 2005)

15 Zhang, Ch.S., Mao, Y. 'Effects of cross section of mine tunnel on the propagation characteristics of UHF radio wave Antennas'. Proc. Seventh Int. Symp. on Propagation and EM Theory, ISAPE'06, Guilin, China, October 2006, pp. 1-5

16 Dudley, D.G., Lienard, M., Mahmoud, S.F., Degauque, P.: 'Wireless propagation in tunnels', IEEE Antennas Propag. Mag., 2007, 49, (2), pp. $11-26$ 